\title{
Impacto del cambio climático en la distribución potencial de tres cultivos agrícolas en México
}

Climate change impact on the potential distribution of three crops in Mexico

\author{
Daniela A. Rivera-Aguirre ${ }^{1}$ (D) , Miguel A. Ortiz- Acosta ${ }^{2}$ (D). Héctor Bernal-Mendoza ${ }^{1,3}$ \\ Gerardo Sánchez-Rojas $^{4}$ (D) , O. Eric Ramírez-Bravo ${ }^{2}$ (D). Daniel Jiménez-García ${ }^{1,2^{*}}$
}
${ }^{1}$ Posgrado en Manejo Sostenible de Agroecosistemas, Instituto de Ciencias, Benemérita Universidad Autónoma de Puebla (BUAP), Edificio Val., Km 1.7 carretera a San Baltazar Tetela, San Pedro Zacachimalpa, 72960, Puebla, Puebla, México. ${ }^{2}$ Laboratorio de Biodiversidad, Centro de Agroecología y Ambiente, Instituto de Ciencias (BUAP).
${ }^{3}$ Complejo Regional Centro (BUAP), Carretera Tecamachalco-Cañada Morelos Km. 7.5, El Salado, 75492, Tecamachalco, Puebla. ${ }^{4}$ Centro de Investigaciones Biológicas, Universidad Autónoma del Estado de Hidalgo, 42186, Pachuca, Hidalgo, México.

*Autor para correspondencia: daniel.jimenez@correo.buap.mx

Fecha de recepción:

20 de enero de 2020

Fecha de aceptación:

23 de marzo de 2021

Disponible en línea:

24 de agosto de 2021

Este es un artículo en acceso

abierto que se distribuye de

acuerdo a los términos de la

licencia Creative Commons.

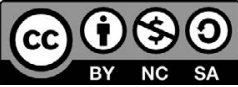

Reconocimiento-

NoComercial-

CompartirIgual 4.0

Internacional

\section{RESUMEN}

Los sistemas agrícolas son sumamente susceptibles ante el cambio climático; sin embargo, poco se sabe de la vulnerabilidad ante este fenómeno de especies nativas o exóticas. En este trabajo, se evaluó el impacto del cambio climático en la distribución potencial de tres especies nativas de México de interés agrícola (algodón, cacahuate, y cacao), a través de modelos de nicho ecológico para 2050. De acuerdo con los 22 modelos de circulación general (GCM), bajo dos Trayectorias de Concentración Representativas (RCP), 4.5 y 8.5, se encontraron incrementos en la distribución potencial de las tres especies. La que presenta mayor incremento es el algodón, y los lugares donde se han encontrado mejores condiciones en el futuro son Tabasco o toda la Península de Yucatán.

\section{PALABRAS CLAVE}

Modelos de nicho ecológico, cultivos agrícolas, susceptibilidad climática.

\section{ABSTRACT}

Agricultural systems are highly susceptible to climate change; however, little is known about the vulnerability of native or exotic species. In this work, we evaluated the impact of climate change on the potential distribution of three species of agricultural interest native to Mexico (cotton, peanut, and cocoa), through ecological niche models looking at the year 2050. According to the 22 General Circulation Models (GCMs) under two Representative Concentration Pathways (RCP), 4.5 and 8.5 , we found increases in the potential distribution of the three species. The species with the greatest increase is cotton, finding conditions in the future in states such as Tabasco or throughout the Yucatan Peninsula.

\section{KEYWORDS}

Ecological niche models, crops, weather susceptibility. 


\section{INTRODUCCIÓN}

El aumento en las emisiones de Gases de Efecto Invernadero (gei), ocurrido a partir del inicio de la Revolución Industrial, provocó un cambio climático global que ha traído como consecuencias modificaciones en la temperatura, mayor variabilidad en las precipitaciones, e intensificación de los eventos climáticos extremos (Vergara et al. 2014). En años recientes, actividades humanas como la quema de combustibles fósiles (Vila 2016), la deforestación (Becerra et al. 2009), la ganadería (Monteny et al. 2001) y la agricultura (Saynes et al. 2016) han intensificado este fenómeno, provocando incrementos mayores en la temperatura del planeta. De acuerdo con Magrin et al. (2007), la temperatura ha aumentado $0.74^{\circ} \mathrm{C}$ durante los últimos 100 años y, para 2100, se proyecta un incremento entre 1.8 a $4.0^{\circ} \mathrm{C}$.

La agricultura de los países en desarrollo se encuentra vulnerable ante los efectos del cambio climático (Müller et al. 2011), ya que depende primordialmente del clima y su variabilidad para el desarrollo de las especies cultivadas (Monterroso-Rivas y Gómez-Díaz 2015). Las predicciones señalan que los efectos del cambio climático se acentuarán durante los siguientes años y se establecerán condiciones extremas para los cultivos (Zarazúa-Villaseñor et al. 2011). En México, los principales afectados serán los productores pequeños, debido a su posición geográfica y a su limitada capacidad adaptativa, lo que pondrá en riesgo la seguridad alimentaria del país (Bellon et al. 2005). En áreas de climas secos, el cambio climático puede inducir a una salinización y desertificación de la tierra agrícola, mientras que, en zonas bajas, el aumento del nivel del mar puede causar severas inundaciones (Magrin et al. 2007).

Las tendencias actuales afirman que el cambio climático es un fenómeno de influencia alta para la expansión y contracción de las especies (Pearson y Dawson 2003), y se prevé que con los efectos de este fenómeno se modificará la producción y distribución de las especies vegetales (Tinoco-Rueda et al. 2011). La distribución de las especies es influenciada por la relación con sus requerimientos agroclimáticos (Lorente et al. 2004); los impactos del cambio climático representan una amenaza para satisfacer estos requerimientos, $y$, por lo tanto, para su presencia y distribución (Jarvis et al.
2008), sus efectos no serán iguales en todas las regiones; por lo tanto, los cultivos se verán afectados negativa o positivamente, de acuerdo con su ubicación geográfica. Sin embargo, se espera que el impacto sea mayor en las zonas áridas y semiáridas (Singh et al. 2014).

Una herramienta útil para evaluar estos riesgos es el Modelado de Nicho Ecológico (MNE) (Peterson et al. 2011), el cual es de gran utilidad en la predicción de los impactos ecológicos y distribuciones potenciales de distintas especies debido al cambio climático (Araújo et al. 2005). Los modelos de nicho ecológico predicen qué tan adecuada es un área para el potencial desarrollo de una especie con respecto a las condiciones ambientales (García 2008).

En el país, se cultiva 15.4 por ciento de todas las especies que constituyen el sistema alimentario mundial (Boege 2009). México pertenece al centro mesoamericano considerado como origen de la agricultura y diversificación de plantas domesticadas (Doebley y Gaut 2006). Entre estas especies se encuentran algunas que son de interés nacional e internacional, como algodón (Gossypium hirsutum L.) (Perales y Aguirre 2008), cacahuate (Arachis hypogaea L.) (Gillier y Silvestre 1970), y cacao (Theobroma cacao L.) (Jaimes y Aránzazu 2010). Estas tres especies representan un ingreso anual de aproximadamente 18 millones de pesos (SIAP 2019), con valor cultural alto (Figueroa-Bautista et al. 2005; Garces 2014; Motamayor et al. 2002) en términos históricos, de identidad y gastronómicos, ya que las tres han sido empleadas en el mundo entero para elaboración de ropa, alimento y chocolate. Es gracias a esta importancia que el objetivo de este trabajo fue evaluar el impacto del cambio climático en la distribución potencial de estas tres especies nativas de interés agrícola en México, con el propósito de identificar las zonas donde se tenderá a aumentar o disminuir su distribución potencial bajo dos escenarios de cambio climático (RCP 4.5 y RCP 8.5) para 2050.

\section{Materiales y Métodos}

\section{Área de estudio}

El territorio mexicano abarca 1,953,162 $\mathrm{km}^{2}$, y en 22 millones de hectáreas del país se llevan a cabo 
actividades agrícolas (11\% del territorio nacional); alrededor de 16.3 millones de hectáreas son de temporal (Monterroso-Rivas y Gómez-Díaz 2015), por lo cual resultan sumamente susceptibles a la variación climática. La agricultura de riego se concentra principalmente en el norte del territorio, mientras que la de temporal se practica más en el sur y centro del país (Ojeda-Bustamante et al. 2012). De acuerdo con el Servicio de Información Agroalimentaria y Pesquera (SIAP 2018), se cultiva algodón en 17,294,082 ha, cacahuate en $21,085,061$ ha y cacao en alrededor de $14,952,889$ ha. Los tres cultivos son representativos de tres regiones climáticas: tropical (Arvelo et al. 2016), subtropical (INIFAP 2002) y seca del territorio mexicano (SAGARPA 2014).

\section{Datos de ocurrencia}

Se obtuvieron datos de los rendimientos de los últimos 15 años para todos los municipios de México de las tres especies, a través del SIAP (2018). Los registros de cacahuate fuera de México no se tomaron en cuenta, ya que no se tiene información de rendimientos y superficie sembrada, que sí obtuvimos para las otras dos especies a nivel municipal. Los municipios seleccionados para determinar la presencia de las especies se determinaron con base en la presencia de al menos 10 años continuos de altos rendimientos de cosecha (una tonelada por encima de cada media anual). Se emplearon los usos del suelo de la Serie V del Instituto Nacional de Estadística y Geografía (INEGI 2013) para colocar dentro de los espacios agrícolas de cada municipio un punto aleatorio, tomando en cuenta las unidades de producción de la Encuesta Nacional Agropecuaria (ENA 2017). Por tanto, de cada municipio se obtuvo un número de puntos al azar dentro de las unidades de producción.

Este proceso se llevó a cabo utilizando el software ArcMap 10.3, con distancia mínima entre cada punto de 7.5 kilómetros.

\section{Variables ambientales}

Los datos ambientales usados fueron las 19 variables bioclimáticas generadas a partir de los valores mensuales de temperatura y precipitación (Cuadro 1), de Worldclim (Hijmans et al. 2005), excluyendo las variables consideradas artefactos espaciales (Escobar et al. 2014), con una resolución espacial de $2.5 \mathrm{~min}$. Con la finalidad de reducir la autocorrelación de las variables y evitar sobreajuste en los modelos (Peterson et al. 2011), se hizo una prueba de Pearson eliminando las variables que tuvieran alto grado de correlación (> 0.8 ). Las variables que se mantuvieron sin correlación se usaron para construir los modelos, mediante el método heurístico empleado por Cobos et al. (2019).

\section{Calibración de los modelos}

Los modelos fueron calibrados utilizando el algoritmo MaxEnt versión 3.3.3k (Phillips et al. 2006), a través del paquete kuenm (Cobos et al. 2019). Se generó la hipótesis del diagrama BAM propuesto por Soberón y Peterson (2005), de acuerdo con el cual las variables deben ser acotadas al área $\mathrm{M}$ o zona de calibración, es decir, la región que ha sido accesible para la especie a través de un tiempo biológico determinado, en este caso, gracias a las condiciones climáticas y de manejo. La zona de calibración se construyó a partir de la intersección de las zonas agrícolas y los límites municipales en los cuales se tenía la presencia de las especies. Con el objetivo de evitar modelos sobreajustados o sumamente complejos, se eligieron los diferentes tipos de parametrización de Maxent (Cuadro 2), para reducir los posibles errores de predictibilidad.

Una vez obtenidos los modelos, fueron evaluados con las pruebas más rigurosas de significancia, rendimiento y complejidad. La significancia de los modelos fue estimada utilizando la prueba de ROC parcial (Peterson et al. 2008), encargada de evaluar la relación entre el error de omisión y la proporción de área predicha como adecuada para la especie; esta prueba es muy importante, pues, además de tener un buen rendimiento, un modelo debe ser significativamente mejor que las predicciones aleatorias (Peterson et al. 2011). Se calcularon las tasas de omisión (OR) para los modelos, al ser la mejor medida para evaluar su rendimiento (Peterson et al. 2011), y se eligieron los modelos con los menores valores de omisión. Finalmente, para elegir los modelos más simples, se utilizó el Criterio de información de Akaike Corregido (AICc), el cual penaliza los modelos con una alta complejidad, al medir el número de parámetros 
Cuadro 1. Variables ambientales utilizadas para crear los modelos de nicho (Hijmans et al. 2005).

\begin{tabular}{ll}
\hline Clave & \\
\hline Bio1 & Temperatura media anual \\
Bio2 & Media mensual *(Max Temp-Min Temp) \\
Bio3 & Isotermalidad (Bio2/Bio7) *(100) \\
Bio4 & Temperatura Estacional (Desviación est. *100) \\
Bio5 & Máxima Temp. del mes más Cálido \\
Bio6 & Mínima Temp. del mes más Frío \\
Bio7 & Temperatura Rango Anual (Bio5-Bio6) \\
Bio8 & Temp Media del Trimestre más Húmedo \\
Bio9 & Temp Media del Trimestre más Seco \\
Bio10 & Temp Media del Trimestre más Cálido \\
Bio11 & Temp Media del Trimestre más Frío \\
Bio12 & Precipitación Media Anual \\
Bio13 & Precipitación del mes más Húmedo \\
Bio14 & Precipitación del mes más Seco \\
Bio15 & Precipitación Estacional \\
Bio16 & Precipitación del Trimestre más Húmedo \\
Bio17 & Precipitación del Trimestre más Seco \\
Bio18 & Precipitación del Trimestre más Cálido \\
Bio19 & Precipitación del Trimestre más Frío \\
\hline
\end{tabular}

en cada uno de ellos, y elige el menos complejo por ser el más fácil de interpretar (Galante et al. 2018); todo este proceso se llevó a cabo en el paquete kuenm (Cobos et al. 2019).

\section{Proyección de los modelos a futuro}

Los modelos seleccionados fueron transferidos al futuro para el año 2050, utilizando los RCPs 4.5 y 8.5 provenientes de 22 Modelos de Circulación General (MCG) (Cuadro 3), obtenidos de Climate Change, Agriculture and Food Security (CCAFS 2018), a una resolución de 2.5 minutos. Finalmente, se combinaron las medianas del presente y las proyecciones futuras de los modelos para obtener los rangos de aumento, disminución o estabilidad en la distribución potencial futura de las especies. Todo el geoprocesamiento se hizo en Arcgis 10.3.

Cuadro 2. Parámetros de los mejores modelos seleccionados con base en las pruebas utilizadas de AICc, Partial- roc y tasas de omisión.

\begin{tabular}{llll}
\hline \multicolumn{1}{c}{ Especie } & \multicolumn{1}{c}{ Característica } & $\begin{array}{c}\text { Multiplicador } \\
\text { de regularización }\end{array}$ & Variable utilizada \\
\hline Gossypium hirsutum & 1, lq, lqp, lqpt, lqpth. & $0.1,0.3,0.5,0.7,1,3,5,7,10$ & Bio2, Bio3, Bio4 \\
Arachys hypogaea & 1, lq, lqp, lqpt, lqpth. & $0.1,0.3,0.5,0.7,1,3,5,7,10$ & Bio1, Bio3, Bio10 \\
Theobroma cacao & 1, lq, lqp, lqpt, lqpth. & $0.1,0.3,0.5,0.7,1,3,5,7,10$ & Bio4, Bio5, Bio16, Bio17 \\
\hline
\end{tabular}


Cuadro 3. MCG utilizados para las proyecciones de los modelos.

\begin{tabular}{|c|c|}
\hline Modelo & Institución creadora del modelo \\
\hline BNU-ESM & College of Global Change and Earth System Science, Beijing Normal University \\
\hline CCMA-CANESM2 & Canadian Centre for Modeling and Analysis \\
\hline CESM1_BGC & $\begin{array}{l}\text { National Science Foundation, Department of Energy, National Center for Atmospheric } \\
\text { Research }\end{array}$ \\
\hline CESM1_CAM5 & $\begin{array}{l}\text { National Science Foundation, Department of Energy, National Center for Atmospheric } \\
\text { Research }\end{array}$ \\
\hline CSIRO_ACCESS1_0 & $\begin{array}{l}\text { CSIRO (Commonwealth Scientific and Industrial Research Organization, Australia) BOM } \\
\text { (Bureau of Meteorology, Australia) }\end{array}$ \\
\hline CSIRO_ACCESS1_3 & $\begin{array}{l}\text { CSIRO (Commonwealth Scientific and Industrial Research Organization, Australia) BOM } \\
\text { (Bureau of Meteorology, Australia) }\end{array}$ \\
\hline GFDL_CM3 & Geophysical Fluid Dinamycs Laboratory \\
\hline GFDL_ESM2G & Geophysical Fluid Dinamycs Laboratory \\
\hline GFDL_ESM2M & Geophysical Fluid Dinamycs Laboratory \\
\hline GISS_E2_H_CC & NASA Goddard Institute for Space Studies \\
\hline GISS_E2_R & NASA Goddard Institute for Space Studies \\
\hline INM_CM4 & Institute for Numerical Mathematics \\
\hline MIROC_ESM_CHEM & $\begin{array}{l}\text { Japan Agency for Marine-Earth Science and Technology, Atmosphere and Ocean Research } \\
\text { Institute (The University of Tokyo) and National Institute for Environmental Studies }\end{array}$ \\
\hline MIROC_ESM & $\begin{array}{l}\text { Japan Agency for Marine-Earth Science and Technology, Atmosphere and Ocean Research } \\
\text { Institute (The University of Tokyo) and National Institute for Environmental Studies }\end{array}$ \\
\hline MIROC_MIROC5 & $\begin{array}{l}\text { Japan Agency for Marine-Earth Science and Technology, Atmosphere and Ocean Research } \\
\text { Institute (The University of Tokyo) and National Institute for Environmental Studies }\end{array}$ \\
\hline MOHC_HADGEM2_CC & Met Office Hadley Centre \\
\hline MOHC_HADGEM2_ES & Met Office Hadley Centre \\
\hline MPI_ESM_LR & Max Planck Institute for Meteorology \\
\hline MRI_CGCM3 & Meteorological Research Institute \\
\hline NCAR_CCSM4 & National Center for Atmospheric Research \\
\hline NCC_NORESM1_M & Norwegian Climate Centre \\
\hline NIMR_HADGEM2_AO & National Institute of Meteorological Research/Korea Meteorological Administration \\
\hline
\end{tabular}

\section{RESUltados Y DISCUSIÓN}

Para cada especie se generaron 1,125 modelos, de los cuales sólo 1 fue significativo para cada especie. Estos presentaron niveles bajos de omisión, lo que indica un desempeño bueno conforme a su capacidad predictiva. De igual manera, son significativamente distintos al azar, como lo demostró la prueba de partial-ROC (Cuadro 4).

Dentro del modelo de cada especie seleccionado como el mejor, el algodón y el cacao tuvieron LQPT, mientras que el mejor modelo de cacahuate tuvo la combinación LQPTH (Cuadro 4). Con respecto a los valores del multiplicador de regularización, las especies de cacahuate y cacao obtuvieron el valor de 1, y el algodón, 3 .

Los modelos de algodón y cacahuate fueron construidos utilizando tres variables bioclimáticas, y cuatro para el caso del cacao. La variable que mejor explicó la presencia del cultivo de algodón fue el rango de temperaturas diurnas con 38 por ciento; mientras que para el cacahuate fue la temperatura media anual con 46 por ciento. Por otra parte, la variable que más información aportó para el cultivo de cacao fue la estacionalidad de la temperatura con 35 por ciento. 
Cuadro 4. Características y parámetros de modelos seleccionados.

\begin{tabular}{|c|c|c|c|c|c|}
\hline Especie & Característica & $\begin{array}{l}\text { Multiplicador } \\
\text { de } \\
\text { regularización }\end{array}$ & AICc & ROC-parcial & Omisión \\
\hline $\begin{array}{l}\text { Gossypium } \\
\text { hirsutum }\end{array}$ & lqpt & 3 & 3938.16 & 0.048 & 0.07 \\
\hline Arachys hypogaea & lqpth & 1 & 608.16 & 0 & 0.36 \\
\hline Theobroma cacao & lqp & 1 & 2519.77 & 0 & 0.02 \\
\hline
\end{tabular}

De acuerdo con las proyecciones a futuro, las zonas de distribución actuales del algodón prevalecerán bajo ambos escenarios (Figura 1), principalmente en la zona norte del país. En ambos escenarios existen incrementos en el nicho en la Península de Yucatán, Tabasco, Veracruz y regiones del Altiplano, de acuerdo con nuestras proyecciones (climas cálido húmedo y templado; Cuadros 5 y 6).

El cacahuate presentará bajo los dos escenarios una gran estabilidad en el nicho (Figura 2), salvo incrementos en el Eje Neovolcánico Transversal, así como en diferentes puntos de la Sierra Madre Occidental y Sierra Madre del Sur (climas templado y semifrío), donde esta especie no se distribuye actualmente. De acuerdo con la transferencia de nuestro modelo al RCP RCP 8.5, algunas regiones de la costa del Pacífico, consideradas inadecuadas, contarán con las características ambientales para
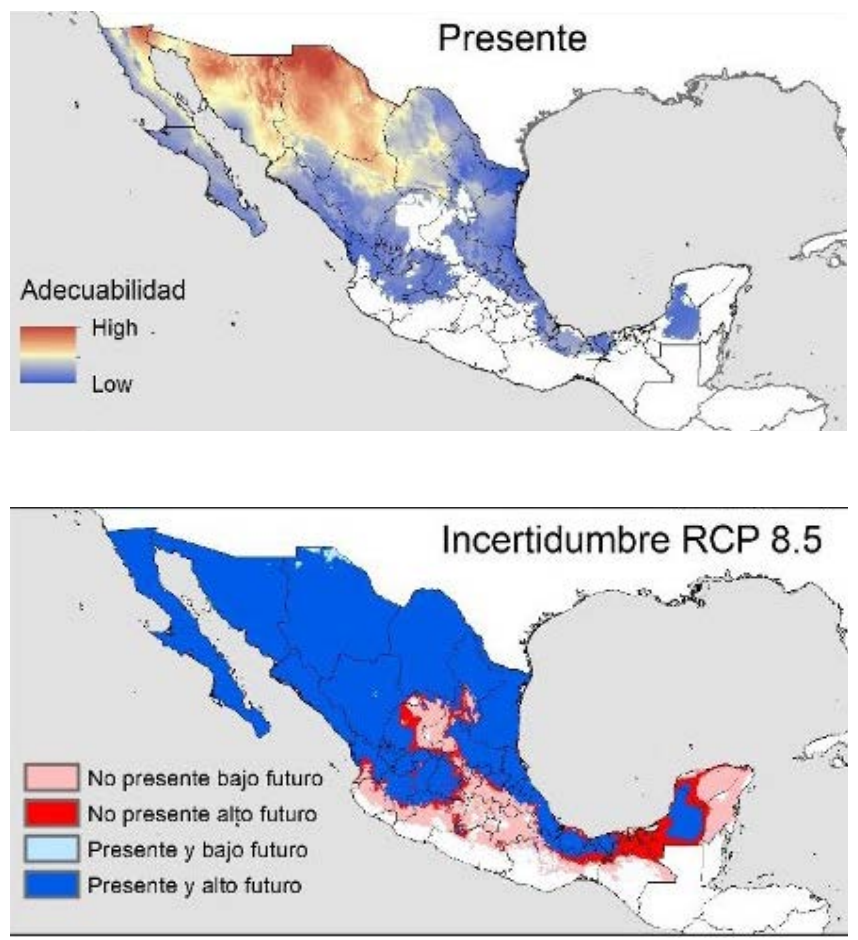

la especie. Sin embargo, en este mismo escenario se predice una reducción de las zonas de distribución actuales del cultivo (de clima cálido) en el Norte de México, en los estados de Tamaulipas y Nuevo León (Figura 2; Cuadros 7 y 8).

El cacao, de acuerdo con nuestros modelos, presentará una prevalencia alta bajo el escenario RCP 4.5; sin embargo, bajo el escenario RCP 8.5 se prevé una prevalencia baja en Tabasco, Chiapas, Veracruz y Oaxaca, donde se produce actualmente el cultivo. Esto puede traer consecuencias principalmente a Chiapas y Tabasco, al ser las entidades productivas más importantes de este cultivo a nivel nacional (SIAP 2014). La Sierra Madre Oriental bajo los dos escenarios presenta mayores superficies de adecuabilidad en el futuro, principalmente en la región del mesófilo de montaña de Puebla, Veracruz e Hidalgo (Figura 3; Cuadros 9 y 10).

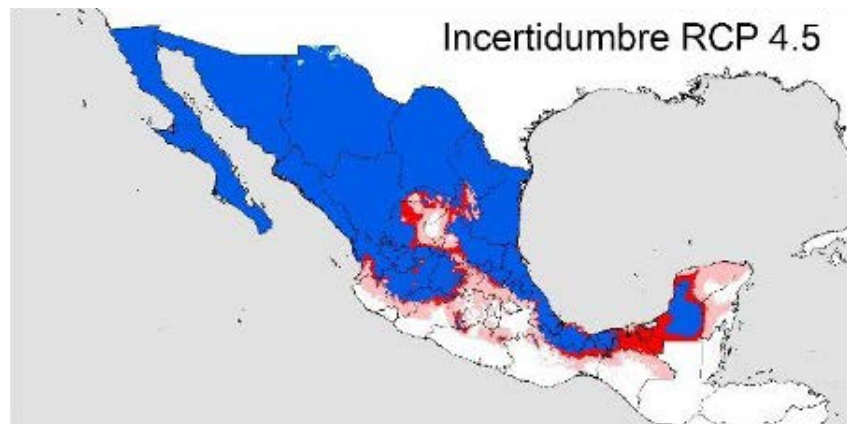

Figura 1. Distribución potencial futura de G. hirsutum, para dos escenarios de emisiones (RCP 4.5 y rcp 8.5) para 2050. En el panel superior se aprecian los niveles de idoneidad ambiental para el presente; se muestran los valores mayores en tonos rojos y los menores en azul. En el panel central e inferior de la figura se muestra la incertidumbre ambiental medida a través de la evaluación de 20 GCMs. El color rosa representa áreas predichas como no aptas para el presente y con pocas probabilidades para el futuro; el color rojo representa áreas predichas como no aptas para el presente, pero con muy altas probabilidades para el futuro; el color azul claro representa áreas predichas como aptas para el presente, pero con pocas probabilidades para el futuro; el color azul oscuro representa áreas predichas como aptas para el presente y con muy altas probabilidades para el futuro, y, el blanco, áreas no aptas para presente y futuro. 
Cuadro 5. Número de hectáreas de cultivo de algodón que tendrán estabilidad, incrementos, pérdidas o no serán adecuadas para 2050 con el RCP 4.5.

\begin{tabular}{|c|c|c|c|c|}
\hline Estado & Estable (ha) & Incremento (ha) & Pérdida (ha) & No Adecuado (ha) \\
\hline Aguascalientes & 533,400 & 58,800 & 6,300 & - \\
\hline Baja California & $8,091,300$ & - & - & - \\
\hline Baja California Sur & $7,746,900$ & - & - & - \\
\hline Campeche & $3,343,200$ & $2,307,900$ & - & - \\
\hline Chiapas & 149,100 & 848,400 & - & - \\
\hline Chihuahua & $26,968,200$ & - & 627,900 & - \\
\hline Coahuila & 16,678200 & - & - & - \\
\hline Colima & - & - & - & - \\
\hline CDMX & - & - & - & - \\
\hline Durango & $13,034,700$ & 105,000 & - & - \\
\hline Guanajuato & $2,253,300$ & 560,700 & 4,200 & - \\
\hline Guerrero & 71,400 & 186,900 & - & - \\
\hline Hidalgo & 756,000 & 205,800 & - & - \\
\hline Jalisco & $4,422,600$ & 462,000 & - & - \\
\hline México & 8,400 & 8,800 & - & - \\
\hline Michoacán & 978,600 & 554,400 & - & - \\
\hline Morelos & - & - & - & - \\
\hline Nayarit & $2,253,300$ & 354,900 & - & - \\
\hline Nuevo León & $5,886,300$ & 579,600 & - & - \\
\hline Oaxaca & 917,700 & 480,900 & - & - \\
\hline Puebla & 588,000 & 56,700 & 6,300 & - \\
\hline Querétaro & 361,200 & 174,300 & - & - \\
\hline Quintana Roo & 29,400 & 321,300 & - & - \\
\hline San Luis Potosí & $3,744,300$ & 842,100 & - & - \\
\hline Sinaloa & $5,926,200$ & - & - & - \\
\hline Sonora & $20,134,800$ & 4,200 & - & - \\
\hline Tabasco & 619,500 & $1,734,600$ & - & - \\
\hline Tamaulipas & $8,156,400$ & 46,200 & - & - \\
\hline Tlaxcala & - & - & - & - \\
\hline Veracruz & $5,928,300$ & 795,900 & - & - \\
\hline Yucatán & 172,200 & 676,200 & - & - \\
\hline Zacatecas & $4,008,900$ & $1,837,500$ & - & - \\
\hline
\end{tabular}

Nuestros modelos de nicho han sido evaluados por las más rigurosas pruebas: 1) significancia, 2) rendimiento y 3) complejidad (Cobos et al. 2019; Peterson et al. 2011; Warren y Seifert 2011). Las transferencias de nuestros modelos a futuro se basaron en la hipótesis BAM (Soberón y Peterson 2005). Nuestro trabajo hace un primer acercamiento a la manera en la que el cambio climático impactará a especies nativas mexicanas de importancia agrícola, que también son fundamentales para la vida productiva del país, además del maíz (Ureta et al. 2011).

De acuerdo con nuestras proyecciones, las tres especies tendrán una persistencia alta del nicho en el futuro, pues se mantendrán la mayor parte de las zonas actuales de distribución (Figuras 1-3); sin embargo, se observa cómo las tres especies cuentan con áreas geográficas nuevas en las que contarán con zonas de adecuabilidad alta distintas a aquellas donde se 

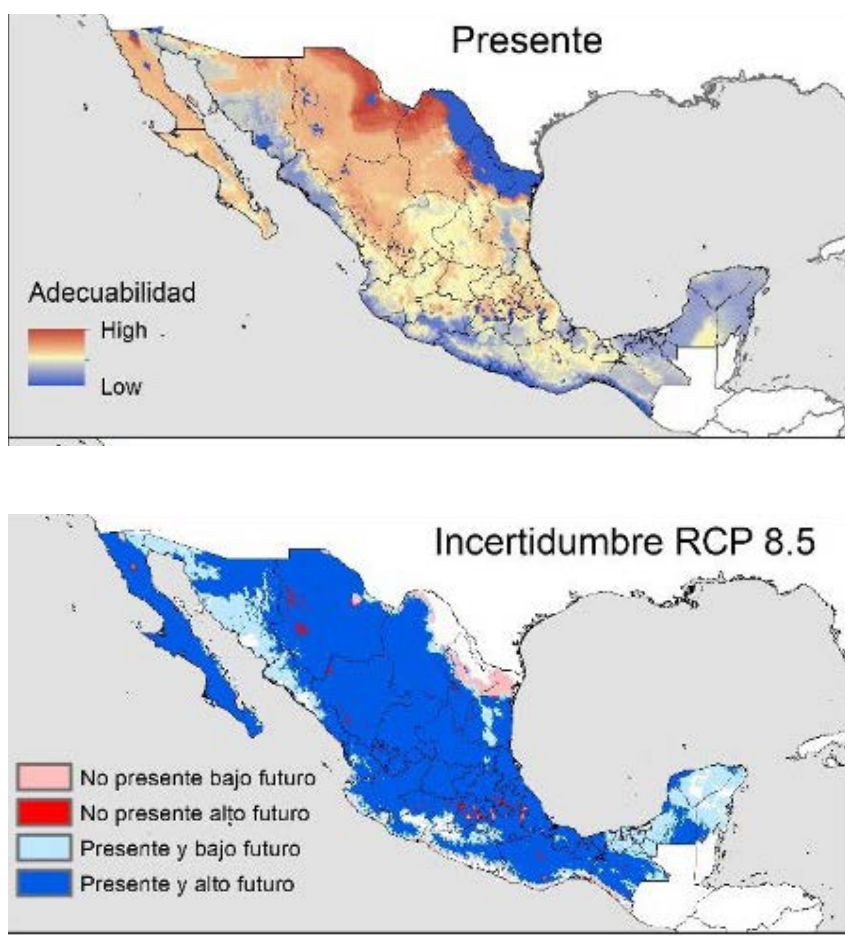

distribuyen actualmente. La plasticidad y tolerancia de las especies agrícolas, generadas a través del manejo, permite que puedan modificar su distribución geográfica potencial y aumentar su tolerancia hacia diversas alteraciones ambientales (Reyes y Martínez 2001). De acuerdo con Chávez-Servia et al. (2011), son las variedades y especies locales las que presentan
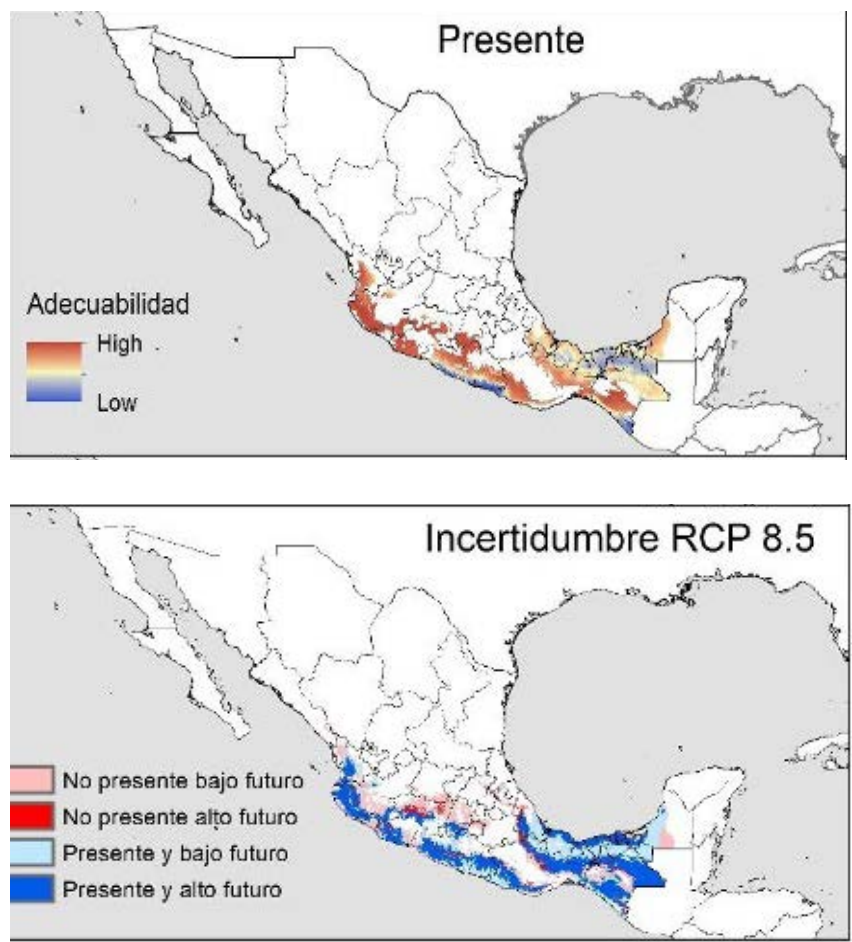

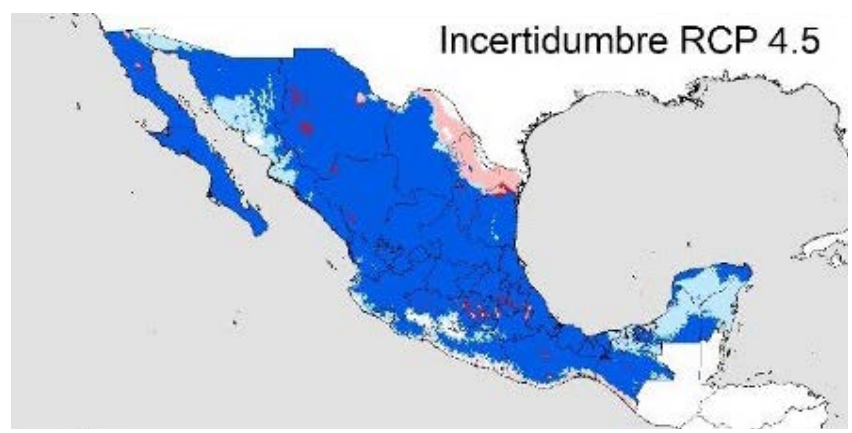

Figura 2. Distribución potencial futura de A. hypogaea, para dos escenarios de emisiones (RCP 4.5 y RCP 8.5) para 2050. En el panel superior se aprecian los niveles de idoneidad ambiental para el presente; se muestran los valores mayores en tonos rojos y los menores en azul. En el panel central e inferior de la figura se muestra la incertidumbre ambiental medida a través de la evaluación de 20 GCMs. El color rosa representa áreas predichas como no aptas para el presente y con pocas probabilidades para el futuro; el color rojo representa áreas predichas como no aptas para el presente, pero con muy altas probabilidades para el futuro; el color azul claro representa áreas predichas como aptas para el presente, pero con pocas probabilidades para el futuro; el color azul oscuro representa áreas predichas como aptas para el presente y con muy altas probabilidades para el futuro, y el blanco, áreas no aptas para presente y futuro.

una mayor plasticidad genética y adaptación ante condiciones ecológicas adversas, $\mathrm{y}$ es dentro de los sistemas de producción tradicionales donde se encuentra la mayor diversidad agrícola nativa, expuesta a distintos procesos de selección natural y artificial, la cual generalmente se encuentra adaptada a nichos particulares (Ríos-Osorio et al. 2014).

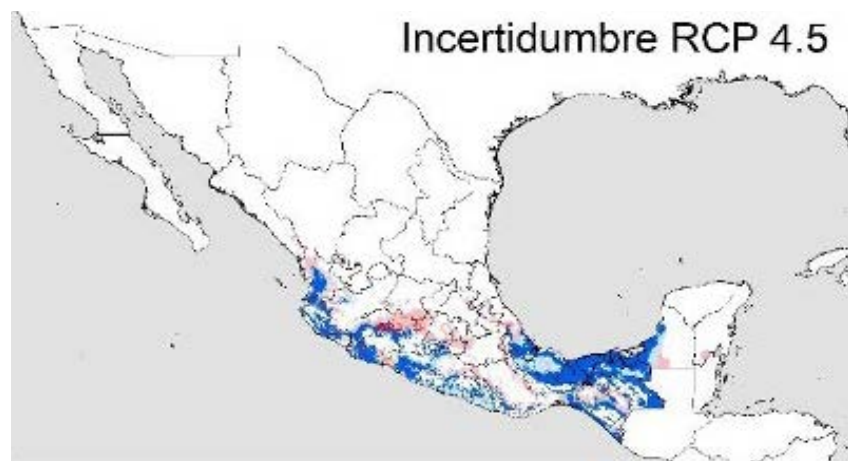

Figura 3. Distribución potencial futura de T. cacao, para dos escenarios de emisiones (RCP 4.5 y RCP 8.5), para 2050. En el panel superior se aprecian los niveles de idoneidad ambiental para el presente; se muestran los valores mayores en tonos rojos y los menores en azul. En el panel central e inferior de la figura se muestra la incertidumbre ambiental medida a través de la evaluación de 20 GCMs. El color rosa representa áreas predichas como no aptas para el presente y con pocas probabilidades para el futuro; el color rojo representa áreas predichas como no aptas para el presente, pero con muy altas probabilidades para el futuro; el color azul claro representa áreas predichas como aptas para el presente, pero con pocas probabilidades para el futuro; el color azul oscuro representa áreas predichas como aptas para el presente y con muy altas probabilidades para el futuro, y el blanco, áreas no aptas para presente y futuro. 
Cuadro 6. Número de hectáreas de cultivo de algodón que tendrán estabilidad, incrementos, pérdidas o no serán adecuadas para 2050 con el RCP 8.5.

\begin{tabular}{|c|c|c|c|c|}
\hline Estado & Estable (ha) & Incremento (ha) & Pérdida (ha) & No Adecuado (ha) \\
\hline Aguascalientes & 533,400 & 58,800 & - & - \\
\hline Baja California & $8,091,300$ & - & - & - \\
\hline Baja California Sur & $7,746,900$ & - & - & - \\
\hline Campeche & $3,343,200$ & $2,343,600$ & - & - \\
\hline Chiapas & 149,100 & $1,129,800$ & - & - \\
\hline Chihuahua & $26,861,100$ & - & 735,000 & - \\
\hline Coahuila & $16,678,200$ & 31,500 & - & - \\
\hline Colima & - & - & - & - \\
\hline CDMX & - & - & - & - \\
\hline Durango & $13,017,900$ & 105,000 & 16,800 & - \\
\hline Guanajuato & $2,257,500$ & 571,200 & - & - \\
\hline Guerrero & 71,400 & 262,500 & - & - \\
\hline Hidalgo & 756,000 & 222,600 & - & - \\
\hline Jalisco & $4,424,700$ & 543,900 & - & - \\
\hline México & - & 88,200 & - & - \\
\hline Michoacán & 978,600 & 672,000 & - & - \\
\hline Morelos & - & - & - & - \\
\hline Nayarit & $2,253,300$ & 378,000 & - & - \\
\hline Nuevo León & $5,886,300$ & 569,100 & - & - \\
\hline Oaxaca & 917,700 & 634,200 & - & - \\
\hline Puebla & 590,100 & 48,300 & 4,200 & - \\
\hline Querétaro & 361,200 & 224,700 & - & - \\
\hline Quintana Roo & 29,400 & 485,100 & - & - \\
\hline San Luis Potosí & $3,744,300$ & 774,900 & - & - \\
\hline Sinaloa & $5,926,200$ & - & - & - \\
\hline Sonora & $20,130,600$ & 8,400 & - & - \\
\hline Tabasco & 619,500 & $1,789,200$ & - & - \\
\hline Tamaulipas & $8,156,400$ & 56,700 & - & - \\
\hline Tlaxcala & - & - & - & - \\
\hline Veracruz & $5,907,300$ & 926,100 & - & - \\
\hline Yucatán & 172,200 & $1,144,500$ & - & - \\
\hline Zacatecas & $4,008,900$ & $1,541,400$ & - & - \\
\hline
\end{tabular}

Nuestros resultados coinciden con Ramírez-Ojeda et al. (2014), al afirmar que los efectos del cambio climático beneficiarán la distribución potencial del algodón, la cual aumentará hacia zonas áridas y semiáridas, debido a su rango climático amplio (-0.1 a $42^{\circ} \mathrm{C}$ ). De acuerdo con las proyecciones de nuestros modelos, la especie tiene una probabilidad alta de distribución futura en los sitios de clima árido y semiárido donde se encuentra actualmente. Se ha reportado que durante 1961 y 2010 los rendimientos de la producción de algodón han incrementado 13 por ciento con el aumento de $1^{\circ} \mathrm{C}$ de temperatura (Chen et al. 2015).

El cultivo de cacahuate se encuentra en regiones de tipo tropical y semiárido, con temperaturas altas y precipitaciones abundantes (Sogut et al. 2016). Los resultados de nuestro trabajo indican que, a pesar de las modificaciones reflejadas por cambio climático, el cacahuate mantendrá los sitios de distribución actual. Sin embargo, las proyecciones muestran también que 
Cuadro 7. Número de hectáreas de cultivo de cacahuate que tendrán estabilidad, incrementos, pérdidas o no será adecuadas para 2050 con el RCP 4.5.

\begin{tabular}{|c|c|c|c|c|}
\hline Estado & Estable (ha) & Incremento (ha) & Pérdida (ha) & No Adecuado (ha) \\
\hline Aguascalientes & 598,500 & - & - & - \\
\hline Baja California & $7,268,100$ & 90,300 & 485,100 & - \\
\hline Baja California Sur & $7,652,400$ & - & 94,500 & - \\
\hline Campeche & $2,587,200$ & - & $3,215,100$ & - \\
\hline Chiapas & $5,796,021$ & 25,200 & 991,200 & - \\
\hline Chihuahua & $26,472,600$ & 543,900 & 359,100 & - \\
\hline Coahuila & $12,131,700$ & - & 999,600 & - \\
\hline Colima & 199,500 & - & 287,700 & - \\
\hline CDMX & 98,700 & 35,700 & - & - \\
\hline Durango & $13,164,900$ & 157,500 & - & - \\
\hline Guanajuato & $3,208,800$ & - & - & - \\
\hline Guerrero & $3,381,000$ & 2,100 & $1,295,700$ & - \\
\hline Hidalgo & $2,133,600$ & 56,700 & - & - \\
\hline Jalisco & $7,303,800$ & 4,200 & 70,700 & - \\
\hline México & $1,860,600$ & 258,300 & 65,100 & - \\
\hline Michoacán & $4,074,000$ & 90,300 & 831,600 & - \\
\hline Morelos & 411,600 & - & 84,000 & - \\
\hline Nayarit & $2,755,200$ & - & 178,500 & - \\
\hline Nuevo León & $3,154,200$ & 39,900 & 336,000 & - \\
\hline Oaxaca & $7,658,700$ & 102,900 & 703,500 & - \\
\hline Puebla & $3,175,200$ & 174,300 & 126,00 & - \\
\hline Querétaro & $1,220,100$ & 2,100 & - & - \\
\hline Quintana roo & $1,495,200$ & - & $2,919,000$ & - \\
\hline San Luis Potosí & $6,510,000$ & - & - & - \\
\hline Sinaloa & 45,060 & - & $1,419,600$ & - \\
\hline Sonora & $12,986,400$ & - & $6,600,300$ & - \\
\hline Tabasco & $1,056,300$ & - & $1,488,900$ & - \\
\hline Tamaulipas & $5,678,400$ & 252,000 & 96,600 & - \\
\hline Tlaxcala & 352,800 & 58,800 & - & - \\
\hline Veracruz & $7,278,600$ & 54,600 & - & - \\
\hline Yucatán & $1,344,000$ & - & $2,809,800$ & - \\
\hline Zacatecas & $8,034,600$ & - & - & - \\
\hline
\end{tabular}

la especie encontrará las condiciones ambientales aptas para ampliar su distribución potencial en zonas templadas de México (Figura 2). Los incrementos en la temperatura pueden afectar el rendimiento de este cultivo (Ketring 1984; Wheeler et al. 1997). Ullaury et al. (2004) consideran que, a pesar de que el cacahuate necesita ciertas condiciones ambientales para su producción, es adaptable a diferentes regiones ambientales. Se sabe que con un descenso de temperaturas aumentaría el periodo de crecimiento del cultivo, pasando de 151 días a una temperatura de $23^{\circ} \mathrm{C}$, a 176 días con temperaturas de $18^{\circ} \mathrm{C}$ (Williams et al. 1975).

La temperatura promedio anual para la producción de cacao es de $25^{\circ} \mathrm{C}$ (entre 23 y $32^{\circ} \mathrm{C}$ ) (Paredes 2004), presente en el Sureste y Península de Yucatán. De acuerdo con nuestros resultados, la distribución potencial del cultivo presenta disminuciones bajo los dos escenarios, con mayores pérdidas de superficie bajo el RCP 8.5 (Figura 3). Los incrementos en ade- 
Cuadro 8. Número de hectáreas de cultivo de cacahuate que tendrán estabilidad, incrementos, pérdidas o no serán adecuadas para 2050 con el RCP 8.5.

\begin{tabular}{|c|c|c|c|c|}
\hline Estado & Estable (ha) & Incremento (ha) & Pérdida (ha) & No Adecuado (ha) \\
\hline Aguascalientes & 598,500 & - & - & - \\
\hline Baja California & $7,016,100$ & 75,600 & 737,100 & - \\
\hline Baja California Sur & $7,389,900$ & - & 357,000 & - \\
\hline Campeche & $2,427,600$ & - & $3,374,700$ & - \\
\hline Chiapas & $5,023,200$ & 27,300 & $1,764,000$ & - \\
\hline Chihuahua & $26,292,000$ & 525,000 & 359,700 & - \\
\hline Coahuila & $12,037,200$ & 35,700 & $10,014,100$ & - \\
\hline Colima & 153,300 & - & 333,900 & - \\
\hline CDMX & 98,700 & 48,300 & - & - \\
\hline Durango & $13,150,200$ & 157,500 & 14,700 & - \\
\hline Guanajuato & $3,208,800$ & - & - & - \\
\hline Guerrero & $2,940,000$ & - & $1,736,700$ & - \\
\hline Hidalgo & $2,133,600$ & - & 56,700 & - \\
\hline Jalisco & $7,106,400$ & 8,400 & 905,100 & - \\
\hline México & $1,829,100$ & 296,100 & 105,100 & - \\
\hline Michoacán & $3,830,400$ & 98,700 & $1,075,200$ & - \\
\hline Morelos & 268,800 & 23,100 & 226,800 & - \\
\hline Nayarit & $2,496,900$ & - & 436,800 & - \\
\hline Nuevo León & $2,872,800$ & 37,800 & 617,400 & - \\
\hline Oaxaca & $7,461,300$ & 111,300 & 900,900 & - \\
\hline Puebla & $2,982,000$ & 195,300 & 319,200 & - \\
\hline Querétaro & $1,220,100$ & 2,100 & - & - \\
\hline Quintana roo & 592,200 & 2100 & - & - \\
\hline San Luis Potosí & $6,426,000$ & - & 84,000 & - \\
\hline Sinaloa & $2,782,500$ & - & $3,143,700$ & - \\
\hline Sonora & $9,607,500$ & - & $9,979,200$ & - \\
\hline Tabasco & 627,900 & - & $1,917,300$ & - \\
\hline Tamaulipas & $4,431,000$ & 44,100 & $1,344,000$ & - \\
\hline Tlaxcala & 352,800 & 65,100 & - & - \\
\hline Veracruz & $7,024,500$ & 58,800 & 254,100 & - \\
\hline Yucatán & 583,800 & - & 35,700 & - \\
\hline Zacatecas & - & - & - & - \\
\hline
\end{tabular}

cuabilidad se encuentran en las zonas con temperaturas semicálidas y templadas. De acuerdo con Paredes (2004) y Enríquez (2006), la actividad de las raíces del cacao empieza a disminuir por debajo de $\operatorname{los} 15^{\circ} \mathrm{C}$ y se recomienda que no se lleve a cabo su cultivo comercial en sitios con estas temperaturas, pues la planta puede empezar a sufrir daños como marchitez extrema (Dostert et al. 2012). Con respecto a los requerimientos de precipitación, la especie se desarrolla idealmente con 1,600 a $2,500 \mathrm{~mm}$ anuales (Paredes 2004). Sin embargo, se ha reportado que este cultivo puede establecerse en áreas secas y en zonas muy contrastantes con su lugar de origen (Carr y Lockwood 2011; Leite et al. 2012), áreas similares a las predichas por el modelo en la costa de Pacífico y Sierra Madre Oriental.

\section{Conclusiones}

Como se demuestra en este estudio, el modelado de nicho ecológico es un método de gran utilidad para 
Cuadro 9. Número de hectáreas de cultivo de cacao que tendrán estabilidad, incrementos, pérdidas o no serán adecuadas para 2050 con el RCP 4.5.

\begin{tabular}{|c|c|c|c|c|}
\hline Estado & Estable (ha) & Incremento (ha) & Pérdida (ha) & No Adecuado (ha) \\
\hline Aguascalientes & - & - & - & - \\
\hline Baja California & - & - & - & - \\
\hline $\begin{array}{l}\text { Baja California } \\
\text { Sur }\end{array}$ & - & - & - & - \\
\hline Campeche & 886,200 & - & 829,500 & - \\
\hline Chiapas & $5,187,000$ & 147,00 & 653,100 & - \\
\hline Chihuahua & - & - & - & - \\
\hline Coahuila & - & - & - & - \\
\hline Colima & 168,000 & 6,300 & 119,700 & - \\
\hline CDMX & - & 4,200 & - & - \\
\hline Durango & - & - & - & - \\
\hline Guanajuato & - & - & - & - \\
\hline Guerrero & $2,895,900$ & 102,900 & $1,457,400$ & - \\
\hline Hidalgo & 10,500 & - & 10,500 & - \\
\hline Jalisco & $1,948,800$ & 69,300 & 378,000 & - \\
\hline México & 338,100 & 48,300 & 153,300 & - \\
\hline Michoacán & $1,497,300$ & 369,600 & 489,300 & - \\
\hline Morelos & 12,600 & 12,600 & 31,500 & - \\
\hline Nayarit & 661,500 & - & 294,000 & - \\
\hline Nuevo León & - & - & - & - \\
\hline Oaxaca & $3,559,500$ & 287,700 & $1,127,700$ & - \\
\hline Puebla & 52,500 & 96,600 & 37,800 & - \\
\hline Querétaro & - & - & 14,700 & - \\
\hline Quintana Roo & - & - & - & - \\
\hline San Luis Potosí & - & - & - & - \\
\hline Sinaloa & - & - & - & - \\
\hline Sonora & - & - & - & - \\
\hline Tabasco & $2,200,800$ & - & 340,200 & - \\
\hline Tamaulipas & - & - & - & - \\
\hline Tlaxcala & - & - & - & - \\
\hline Veracruz & $2,631,300$ & 142,800 & $1,236,900$ & - \\
\hline Yucatán & - & - & - & - \\
\hline Zacatecas & - & - & - & - \\
\hline
\end{tabular}

crear estrategias adecuadas que permitan asegurar una buena producción de alimentos en el futuro, al tener un mejor y más acertado manejo de las especies. Este trabajo es una herramienta a través de la cual productores y distintos organismos pueden generar acciones de mitigación y adaptación ante cambio climático, con la finalidad de no ver reducidos sus ingresos económicos y poder favorecer la seguridad alimentaria.
Nuestro trabajo presenta una visión de tres cultivos con orígenes climáticos diferentes, con pérdidas y ganancias de adecuabilidad. Los tres cultivos son persistentes; sin embargo, muestran rasgos de incrementos en la disponibilidad ambiental para zonas más altas (Sierra Madre Oriental y Sierra Madre Occidental para el caso del cacao; eje Neovolcánico Transversal y Sierra Madre Occidental para el caso del cacahuate) y zonas más secas para el caso del algodón 
Cuadro 10. Número de hectáreas de cultivo de cacao que tendrán estabilidad, incrementos, pérdidas o no serán adecuadas para 2050 con el RCP 8.5.

\begin{tabular}{|c|c|c|c|c|}
\hline Estado & Estable (ha) & Incremento (ha) & Pérdida (ha) & No Adecuado (ha) \\
\hline Aguascalientes & - & - & - & - \\
\hline Baja California & - & - & - & - \\
\hline $\begin{array}{l}\text { Baja California } \\
\text { Sur }\end{array}$ & - & - & - & - \\
\hline Campeche & 182,700 & - & $1,533,000$ & - \\
\hline Chiapas & $4,053,000$ & 132,300 & $1,787,100$ & - \\
\hline Chihuahua & - & - & - & - \\
\hline Coahuila & - & - & - & - \\
\hline Colima & 193,200 & 2,100 & 94,500 & - \\
\hline CDMX & - & - & - & - \\
\hline Durango & - & - & - & - \\
\hline Guanajuato & - & - & - & - \\
\hline Guerrero & $2,509,500$ & 121,800 & $1,843,800$ & - \\
\hline Hidalgo & 8,400 & 16,800 & 12,600 & - \\
\hline Jalisco & $1,730,400$ & 73,500 & 596,400 & - \\
\hline México & 291,900 & 50,400 & 199,500 & - \\
\hline Michoacán & $1,281,000$ & 411,600 & 705,600 & - \\
\hline Morelos & 8,400 & 18,900 & 35,700 & - \\
\hline Nayarit & 510,300 & - & 445,200 & - \\
\hline Nuevo León & - & - & - & - \\
\hline Oaxaca & $2,954,700$ & 327,600 & $1,732,500$ & - \\
\hline Puebla & 50,400 & 39,900 & 113,400 & - \\
\hline Querétaro & - & - & 14,700 & - \\
\hline Quintana Roo & - & - & - & - \\
\hline San Luis Potosí & - & - & - & - \\
\hline Sinaloa & - & - & - & - \\
\hline Sonora & - & - & - & - \\
\hline Tabasco & $1,043,700$ & - & $1,447,300$ & - \\
\hline Tamaulipas & - & - & - & - \\
\hline Tlaxcala & - & - & - & - \\
\hline Veracruz & $1,373,400$ & 147,000 & $2,494,800$ & - \\
\hline Yucatán & - & - & - & - \\
\hline Zacatecas & - & - & - & - \\
\hline
\end{tabular}

(Altiplano Potosino-Zacatecano) y regiones húmedas (Tabasco y Península de Yucatán). La principal limitante de nuestros modelos es la integración del manejo en los diferentes cultivos; sin embargo, las especies de interés agrícola cuentan con una fuerte dependencia de las condiciones climáticas que se ven reflejadas claramente por las ocurrencias.

\section{Agradecimientos}

Al Consejo Nacional de Ciencia y Tecnología y a la Benemérita Universidad Autónoma de Puebla por el apoyo para llevar a cabo esta investigación. 


\section{Literatura Citada}

Araújo MB, Pearson RG, Thuillers W, Erhard M. 2005. Validation of species-climate impact models under climate change. Global Change Biology 11: 1504-1513. https://doi.org/10.1111/j.1365-2486.2005.001000.x

Arvelo M, Delgado T, Maroto S, Rivera J, Higuera I, Navarro A. 2016. Estado actual sobre la producción y el comercio del cacao en América. Folleto técnico. Instituto Interamericano de Cooperación para la Agricultura. San José, Costa Rica.

Becerra M, Mance H, Barrera X, García C. 2009. Cambio climático: lo que está en juego. Boletín divulgativo. Universidad de Los Andes. Bogotá, Colombia.

Bellon MR, Hodson D, Bergvinson D, Beck D, MartinezRomero E, Montoya Y. 2005. Targeting agricultural research to benefit poor farmers: Relating poverty mapping to maize environments in Mexico. Food Policy 30: 476-492. https://doi.org/10.1016/j. foodpol.2005.09.003

Boege E. 2009. Centros de origen, pueblos indígenas y diversificación del maíz. Revista de Cultura Científica 92: 18-28.

Carr MKV, Lockwood G. 2011. The water relations and irrigation requirements of cocoa (Theobroma cacao L.): A review. Experimental Agriculture 47: 653-676. https://doi.org/10.1017/S0014479711000421

[CCFAS] Climate Change, Agriculture and Food Security. [internet]. 2018. GCM Downscaled Data Porta. [citado 2019 sept 22]. Disponible en: http://www.ccafs-clima te.org/data_spati al_downscaling/

Chávez-Servia JL, Carrillo J, Vera A, Rodríguez E, Lobato R. 2011. Uso actual y potencial del jitomate silvestre mexicano. Secretaría de Agricultura, Desarrollo Rural, Ganadería, Pesca y Alimentación. Oaxaca, México.

Chen C, Pang Y, Pan X, Zhang L. 2015. Impacts of climate change on cotton yield in China from 1961 to 2010 based on provincial data. Journal of Meteorological Research 29: 515-524. https://doi.org/10.1007/ s13351-014-4082-7

Cobos ME, Peterson AT, Osorio-Olvera L, Jiménez-García D. 2019. An exhaustive analysis of heuristic methods for variable selection in ecological niche modeling and species distribution modeling. Ecological Informatics 53: 100983. https://doi.org/10.1016/j. ecoinf.2019.100983
Doebley J, Gaut B. 2006. The molecular genetics of crop domestication. Cell 127: 1309-1321. https://doi. org/10.1016/j.cell.2006.12.006

Dostert N, Roque GJ, Echeverría A, Torre M, Weigend M. 2012. Hoja botánica: Cacao- Theobroma cacao L. Folleto Técnico D38/08-19. Programa Desarrollo Rural Sostenible. Lima, Perú.

[ENA] Encuesta Nacional Agropecuaria. [internet]. 2017. Presentación de resultados 2017. [citado 2019 ag 21]. Disponible en: https://www.inegi.org.mx/programas/ ena/2019.

Enríquez GA. 2006. Fenología y fisiología del cacao. Seminario Taller Internacional, Producción, Calidad y Mercadeo de Cacaos Especiales. Quevedo, Ecuador.

Escobar LE, Lira-Noriega A, Medina-Vogel G, Peterson AT. 2014. Potential for spread of the white-nose fungus (Pseudogymnoascus destructans) in the Americas: Use of Maxent and NicheA to assure strict model transference. Geospatial Health 9: 221-229. https:// doi.org/10.4081/gh.2014.19

Figueroa-Bautista P, Gerritsen PRW, Villalvazo-López VM, Cruz-Sandoval G. 2005. Articulando la sostenibilidad ecológica, económica y social: el caso del cacahuate orgánico. Economía, Sociedad y Territorio 19: 477-497.

Galante PJ, Alade B, Muscarella R, Jansa SA, Goodman SM, Anderson RP. 2018. The challenge of modeling niches and distributions for data-poor species: A comprehensive approach to model complexity. Ecography 41: 726-736. https://doi.org/10.1111/ ecog.02909

Garces N. 2014. Antecedentes y condiciones actuales sobre el manejo y usos del algodón en seis comunidades del Totonacapan, Veracruz. Tesis de Maestría en Ciencias. Universidad Veracruzana. Xalapa, México.

García MR. 2008. Modelos predictivos de riqueza de diversidad vegetal, comparación y optimización de métodos de modelado ecológico. Tesis de Doctorado. Universidad Complutense de Madrid. Madrid, España.

Gillier P, Silvestre P. 1970. El cacahuate o maní. Blume. Zaragoza, España.

Hijmans RJ, Cameron SE, Parra JL, Jones PG, Jarvis A. 2005. Very high resolution interpolated climate surfaces for global land areas. International Journal of Climatology 25: 1965-1978. https://doi. org/10.1002/joc. 1276 
[INEGI] Instituto Nacional de Geografía y Estadística. [internet]. 2013. Uso de suelo y vegetación, escala 1: 250 000, serie V (capa unión), escala 1:250000. México. [citado 2019 sept 20]. Disponible en: https://www.inegi.org.mx/temas/usosuelo/

[INIFAP] Instituto Nacional de Investigaciones Forestales Agrícolas y Pecuarias. [internet]. 2002. Producción del cultivo de cacahuate en el estado de Morelos. Folleto Técnico 18. Zacatepec, Morelos. [citado 2019 sept 21]. Disponible en: https://docplayer. es/39327979-Produccion-del-cultivo-de-cacahuateen-el-estado-de-morelos.html

Jaimes Y, Aránzazu F. 2010. Manejo de las enfermedades del cacao (Theobroma cacao L.) en Colombia con énfasis en Monilia (Moniliophtora roreri). Manual técnico. Corporación Colombiana de Investigación Agropecuaria. Colombia.

Jarvis A, Lane A, Hijmans RJ. 2008. The effect of climate change on crop wild relatives. Agriculture, Ecosystems and Environment 126: 13-23. https://doi. org/10.1016/j.agee.2008.01.013

Ketring D. 1984. Temperature effects on vegetative and reproductive development of peanut. Crop Science 24: 877-882.

Leite VJB, Fonseca VE, Sodré AG, Valle RR, Nascimento NM, Marrocos LPC. 2012. Comportamento produtivo de cacau no semiárido do Brasil. Agrotrópica 24: 85-90. https://doi.org/10.21757/0103-3816.2012V24N2P85-90

Lorente I, Gamo D, Gómez JL, Santos R, Flores L, Camacho A, Navarro J. 2004. Los efectos biológicos del cambio climático. Ecosistemas 13: 103-110.

Magrin G, Gay C, Cruz D, Giménez G, Moreno A, Nagy G, Nobre C, Villamizar A. 2007. Latin America. Climate change 2007: Impacts, adaptation and vulnerability. En: Campos M, Alcarez E, Fearnside P, editores. Contribution of Working Group II to the Fourth Assesment Report of the Intergovernmental Panel on Climate Change. Cambridge, Cambridge University Press. P. 581-615.

Monteny GJ, Groenestein CM, Hilhorst MA. 2001. Interactions and coupling between emissions of methane and nitrous oxide from animal husbandry. Nutrient Cycling in Agroecosystems 60: 123-132. https://doi. org/10.1023/A:1012602911339

Monterroso-Rivas I, Gómez-Díaz JD. 2015. Sistemas de Producción de Alimentos y Seguridad Alimentaria. En: Gay C, Rueda-Abad J, editores. Reporte Mexicano de
Cambio Climático. Grupo II: Impactos, Vulnerabilidad y Adaptación. México, UNAM. P. 97-119z.

Motamayor JC, Risterucci AM, López PA, Ortiz CF, Moreno A, Lanaud C. 2002. Cacao domestication I. The origin of the cacao cultivated by the Mayas. Heredity 89: 380-386. https://doi.org/10.1038/sj.hdy.6800156

Müller C, Cramer W, Hare WL, Lotze-Campen H. 2011. Climate change risks for African agriculture. Proceedings of the National Academy of Sciences 108: 4313-4315. https://doi.org/10.1073/pnas.1015078108

Ojeda-Bustamante W, Sifuentes-Ibarra E, RojanoAguilar A, Iñiguez-Covarrubias M. 2012. Adaptación de la agricultura de riego ante el cambio climático. En: Polioptro F, Martínez A, Patiño-Gómez C, editores. Adaptación al cambio climático en los recursos hídricos de México. Jiutepec, Morelos. Instituto Tecnológico del Agua. P. 71-110.

Paredes M. 2004. Manual de cultivo del cacao. Ministerio de agricultura. Programa para el desarrollo de la Amazonia (PROAMAZONIA). Lima, Perú.

Pearson RG, Dawson TP. 2003. Predicting the impacts of climate change on the distribution of species: Are bioclimate envelope models useful? Global Ecology and Biogeography 12: 361-371. https://doi. org/10.1046/j.1466822X.2003.00042.x

Perales HR, Aguirre JR. 2008. Biodiversidad humanizada. En: Capital natural de México, vol. I: Conocimiento actual de la biodiversidad. México, CONABIO. P. 565-603.

Peterson AT, Papeş M, Soberón J. 2008. Rethinking receiver operating characteristic analysis applications in ecological niche modeling. Ecological Modelling 213: 63-72. https://doi.org/10.1016/j.ecolmodel.2007.11.008

Peterson AT, Soberón J, Pearson RG, Anderson RP, Martínez-Meyer E, Nakamura M, Araújo MB. 2011. Ecological Niches and Geographic Distributions. Princeton University Press. Nueva Jersey, Estados Unidos.

Phillips SJ, Anderson RP, Schapire RE. 2006. Maximum entropy modeling of species geographic distributions. Ecological Modelling 190: 231-259. https://doi. org/10.1016/j.ecolmodel.2005.03.026

Ramírez-Ojeda G, Ruiz-Corral JA, Pérez-Mendoza C, Villavicencio-García $R$, Mena S, Durán-Puga N. 2014. Impactos del cambio climático en la distribución geográfica de Gossypium hirsutum L. en México. Revista Mexicana de Ciencias Agrícolas 5: 1885-1895. 
Reyes JM, Martínez D. 2001. La plasticidad de las plantas. Elementos: Ciencia y Cultura 8: 39-43.

Ríos-Osorio O, Chávez-Servia JL, Carrillo-Rodríguez JC. 2014. Producción tradicional y diversidad de tomate (Solanum lycopersicum L.) nativo: un estudio de caso en Tehuantepec-Juchitán, México. Agricultura, Sociedad y Desarrollo 11: 35-51.

[SAGARPA] Secretaría de Agricultura, Ganadería, Ganadería, Desarrollo Rural, Pesca y Alimentación. [internet]. 2014. Análisis de la cadena de valor en la producción de algodón en México. [citado 2019 ag 16]. Disponible en: http://www.redinnovagro.in/ pdfs/algodon.pdf

Saynes V, Etchevers JD, Paz F, Alvarado LO. 2016. Emisiones de gases de efecto invernadero en sistemas agrícolas de México. Terra Latinoamericana 34: 83-96.

[SIAP] Servicio de Información Agroalimentaria y Pesquera. [internet]. 2014. Datos abiertos. [citado 2019 ag 18]. Disponible en: http://infosiap.siap.gob.mx/gob$\mathrm{mx} /$ datosAbiertos.php

[SIAP] Servicio de Información Agroalimentaria y Pesquera. [internet]. 2018. Datos abiertos. [citado 2019 ag 19]. Disponible en: http://infosiap.siap.gob.mx/gob$\mathrm{mx} /$ datosAbiertos.php

[SIAP] Servicio de Información Agroalimentaria y Pesquera. [internet]. 2019. Expectativas de producción agropecuaria y pesquera. [citado 2019 ag 19]. Disponible en: https://www.gob.mx/siap/documentos/ expectativas-de-produccion-agropecuaria-pesquera

Singh P, Nedumaran S, Ntare BR, Boote KJ, Singh NP, Srinivas K, Bantilan MCS. 2014. Potential benefits of drought and heat tolerance in groundnut for adaptation to climate change in India and West Africa. Mitigation and Adaptation Strategies for Global Change 19: 509529. https://doi.org/10.1007/s11027-012-9446-7

Soberón J, Peterson AT. 2005. Interpretation of fundamental ecological niches and species' distributional areas. Biodiversity Informatics 2: 1-10. https://doi. org/10.17161/bi.v2i0.4

Sogut T, Ozturk F, Kizil S. 2016. Effect of sowing time on peanut (Arachis hypogaea L.) cultivars: II. Fatty acid composition. Agriculture and Agricultural Science Procedia 10: 76-82. https://doi.org/10.1016/j.aaspro.2016.09.018

Tinoco-Rueda JA, Gómez-Díaz JD, Monterroso-Rivas AI. 2011. Efectos del cambio climático en la distribución potencial del maíz en el estado de Jalisco, México. Terra Latinoamericana 29: 161-168.
Ullaury J, Guzmán R, Alava J. 2004. Guía del cultivo de maní para las zonas de Loja y El Oro. Boletín divulgativo 314. Instituto Nacional de Investigaciones Agropecuarias. Machala, Ecuador.

Ureta C, Martínez-Meyer E, Perales HR, Álvarez-Buylla ER. 2011. Projecting the effects of climate change on the distribution of maize races and their wild relatives in Mexico. Global Change Biology 18: 1073-1082. https:// doi.org/10.1111/j.1365-2486.2011.02607.x

Vergara W, Ríos A, Trapido P, Malarín H. 2014. Agricultura y clima futuro en América Latina y el Caribe. Impactos sistémicos y posibles respuestas. Documento de debate. Banco Interamericano de Desarrollo. Washington DC, Estados Unidos.

Vila M. 2016. Estudio de los cambios en los patrones del oleaje en la costa catalana debido al efecto del cambio climático. Tesis de licenciatura. Escuela Técnica Superior de Ingeniería de Caminos. Barcelona, España.

Warren DL, Seifert SN. 2011. Ecological niche modeling in Maxent: The importance of model complexity and the performance of model selection criteria. Ecological Applications 21: 335-342. https://doi:10.1890/10-1171.1

Wheeler TR, Chatzialioglou A, Craufurd PQ, Ellis RH, Summerfield RJ. 1997. Dry matter partitioning in peanut exposed to high temperature stress. Crop Science 37: 1507-1513. https://doi.org/10.2135/ cropsci1997.0011183X003700050016x

Williams JH, Wilson JHH, Bate GC. 1975. The growth and development of four groundnut (Arachis hypogaea L.) cultivars in Rhodesia. Rhodesian Journal of Agricultural Research 13: 131-144.

Zarazúa-Villaseñor P, Ruiz-Corral JA, González-Eguiarte DR, Flores-López HE, Ron-Parra J. 2011. Impactos del cambio climático sobre la agroclimatología del maíz en Ciénega de Chapala, Jalisco. Revista Mexicana de Ciencias Agrícolas 2: 351-363. 